\title{
Thierry Libert
}

\section{ZF AND THE AXIOM OF CHOICE IN SOME PARACONSISTENT SET THEORIES}

\begin{abstract}
In this paper, we present set theories based upon the paraconsistent logic Pac. We describe two different techniques to construct models of such set theories. The first of these is an adaptation of one used to construct classical models of positive comprehension. The properties of the models obtained in that way give rise to a natural paraconsistent set theory which is presented here. The status of the axiom of choice in that theory is also discussed. The second leads to show that any classical universe of set theory (e.g. a model of $Z F$ ) can be extended to a paraconsistent one, via a term model construction using an adapted bisimulation technique.
\end{abstract}

\section{Introduction}

The ideal calculus, i.e. the first-order axiomatization of naive set theory that consists of the general comprehension schema and the axiom of extensionality, is inconsistent in classical logic. Legitimately, one may tamper rather with the underlying logic than the axiomatization in order to keep our naive conception of set safe from the antinomies that appear in "classical" reasoning, trying to find which logics can support, in a satisfactory way, this naive (yet intuitively correct) conception. Several attempts have been made so far to consider such ideal calculi based upon non-classical logics. For instance, let us just mention [5] and [13] where the consistency of some versions of this calculus was proved respectively in the Lukasiewicz three-valued logic $\mathrm{E}_{3}$ and in the paraconsistent three-valued logic LP. In either (and others as well), a possible objection is the absence of the equality relation in the language and, a fortiori, in the formulae defining sets. We show here that, in Pac, this objection can be overcome in a natural way (of course, others objections are possible). 
Actually, Pac is the name under which the propositional version of this logic appeared in [1], but it had previously appeared in [3], under the name $P I^{S}$, or even in [2] under the name $R M_{3}^{\sim}$; more recently, the predicative version of that logic was studied in [4] under the name CLuNs. This plurality reflects, in a sense, the impression that many logicians treat non-classical logics only as pure calculi without investigating further their interpretations and applications. In this paper, we have tried to tone down this impression by showing there exist natural models for a paraconsistent set theory (in Pac), or if one does not agree on the word "natural", let us say less pathological or artificial than those which are especially constructed just to guarantee the consistency of such non-classical theories (as in $[13],[5],[10]^{1}$, and as in the last section of this paper!).

To make this paper as self-contained as possible and because the logic we use has appeared under different names and aspects in the literature, the next section of this paper is aimed to present it in the context of set theory introducing a syntactical variant of the ideal calculus in that logic. We describe afterwards two different techniques to build non-trivial models.

All the models are constructed in usual set theory, $Z F$ or some suitable extension of it, so that the consistency results proved here are relative to that theory. We shall use the common notations of $Z F$ as well as any set theoretical feature. Beside these we wish to introduce the following ones:

If $R$ is any binary relation (i.e. a set of ordered pairs), $R^{-1}$ will denote $\{(x, y) \mid(y, x) \in R\}$, and for any set $A$, we define $R " A$ as $\{b \mid \exists a \in A: a R b\}$. These notations will be mainly used in the functional case; then, for any function $f$ and any $a$ within the domain of $f$, we define $f^{\prime} a$ by $f^{\prime \prime}\{a\}=\left\{f^{\prime} a\right\}$. So we have, for any subset $A$ of the domain of $f, f^{\prime \prime} A=\left\{f^{\prime} a \mid a \in A\right\}$, and for any subset $B$ of the codomain of $f, f^{-1} " B=\left\{a \mid f^{\prime} a \in B\right\}$. The observant reader will notice that the membership relation in the metatheory is denoted by a small ' $\epsilon$ ' to distinguish it from the big ' $\in$ ' that will appear in the languages of set theories. By the way, if $\varphi$ denotes a formula in one of those languages, then the notation $\varphi\left(x_{1}, \ldots, x_{k}\right)$ will be used as usual for denoting that formula when its free variables are among $x_{1}, \ldots, x_{k}$. In this case, $\varphi\left[a_{1}, \ldots, a_{k}\right]$ will designate the formula obtained from $\varphi$ by substituting $a_{i}$ for each occurrence of the variable $x_{i}$ in $\varphi$, for $i=1, \ldots, k$.

\footnotetext{
1 Actually, in [5] and [10], it is not a paraconsistent logic that is involved but the paracomplete (or "partial") three-valued logic $\mathrm{E}_{3}$.
} 


\section{A paraconsistent set theory}

Because of the nature of Russell's paradox, a paraconsistent logic seems to be especially suitable for our purposes. The one that is used here, Pac, is a three-valued logic. As non-classical logics are not a familiar topic for most set-theorists, we shall briefly survey how a set theory might be presented in that logic. We will content ourselves with presenting the key steps and some illustrative properties of the logic without any proofs, but the reader really should not meet with difficulties in verifying them if he ever tried (some details can be found here and there in the references mentioned in the introduction).

As usual, the language of set theory is the first-order predicate calculus built up from the signature $\mathcal{L}:=\{\in,=\}$ where ' $=$ ' is really meant to be an "equality" relation. In $P a c$, the primitive connectives and quantifiers will be denoted by $\sim, \wedge, \vee, \rightarrow, \forall, \exists$. This logic can be characterized semantically by means of the following valuation system:

- the set of truth values is $V:=\{0, i, 1\}$, ordered by $0<i<1$, and the set of designed ones is $D:=\{i, 1\}$;

- the valuation functions for the primitive connectives and quantifiers are defined as follows:

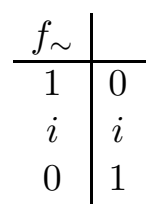

\begin{tabular}{c|ccc}
$f_{\wedge}$ & 1 & $i$ & 0 \\
\hline 1 & 1 & $i$ & 0 \\
$i$ & $i$ & $i$ & 0 \\
0 & 0 & 0 & 0
\end{tabular}

\begin{tabular}{c|ccc}
$f_{\vee}$ & 1 & $i$ & 0 \\
\hline 1 & 1 & 1 & 1 \\
$i$ & 1 & $i$ & $i$ \\
0 & 1 & $i$ & 0
\end{tabular}

\begin{tabular}{c|ccc}
$f_{\rightarrow}$ & 1 & $i$ & 0 \\
\hline 1 & 1 & $i$ & 0 \\
$i$ & 1 & $i$ & 0 \\
0 & 1 & 1 & 1
\end{tabular}

For any $A \subseteq V,\left\{\begin{array}{l}f_{\forall}^{\prime} A:=\min A \\ f_{\exists}^{\prime} A:=\max A\end{array}\right.$

We should emphasize that the paraconsistent logic LP used in [13] is exactly the implicationless fragment of $P a c$. In $L P$, the primitive implication, denoted here by ' $\rightsquigarrow$ ', is in fact definable from ' $~$ ' and ' $V$ ' as in classical logic:

$$
p \rightsquigarrow q: \equiv \sim p \vee q \quad \text { (for any propositional variables } p, q \text { ) }
$$

Notice incidentally that ' $\rightsquigarrow$ ' is weaker than ' $\rightarrow$ ', but ' $\rightsquigarrow$ ' is self-contrapositive whereas ' $\boldsymbol{C}$ ' is not. Its contrapositive will be denoted by:

$$
p \rightarrow q: \equiv \sim q \rightarrow \sim p \quad \text { (for any propositional variables } p, q \text { ) }
$$

and it is used for defining a self-contrapositive implication:

$$
p \Rightarrow q: \equiv(p \rightarrow q) \wedge(p \neg q) \quad(\text { for any propositional variables } p, q)
$$


which is obviously stronger than ' - '. At this point, let us mention that, among all these implication connectives, ' $\longrightarrow$ ' is the only one fulfilling the deduction property: $\Sigma \cup\{\varphi\} \vDash_{P a c} \psi \Leftrightarrow \Sigma \vDash_{P a c} \varphi \rightarrow \psi$, where ' $\vDash_{P a c}$ ' is the consequence relation in $P a c$ that is defined specifically below. The right to left meta-implication ' $\Leftarrow$ ' corresponds actually to modus ponens $(M P)$, while the converse is sometimes referred to as conditionalization $(C)$. In fact, one can show that ' $\rightsquigarrow$ ' fulfills $C$ but not $M P$, whereas ' $\Longrightarrow$ ' satisfies $M P$ but not $C$. The implication connectives derived from ' - ' also lead to syntactically define several bi-implication connectives, as the notations suggest: $\triangleleft, \nabla$, $\rightleftharpoons, \leftrightharpoons, \Leftrightarrow$

An $\mathcal{L}$-structure $\mathcal{M}: \equiv\left\langle M ; \mathcal{L}_{\mathcal{M}}\right\rangle$ for Pac is a non-empty domain of quantification $M$ (the universe of the structure) together with a function $\mathcal{L}_{\mathcal{M}}$ which interprets each (binary) relational symbol $R \in \mathcal{L}$ as a function $R_{\mathcal{M}}$ from $M \times M$ into $V .^{2}$

Then, an $\mathcal{L}$-assignment for Pac consists of a pair $(\mathcal{M}, v)$ where $\mathcal{M}$ is an $\mathcal{L}$-structure and $v$ is a function that assigns an element of $M$ to each variable of the language. Given such an $\mathcal{L}$-assignment we are able to compute the truth value $v_{\mathcal{M}}{ }^{\prime} \varphi$ of any $\mathcal{L}$-formula in $P a c$ : for any $R \in \mathcal{L}, v_{\mathcal{M}}{ }^{\prime}(x R y):=$ $R_{\mathcal{M}}\left(v^{\prime} x, v^{\prime} y\right)$ and $v_{\mathcal{M}}^{\prime} \varphi$ is defined inductively from these as it should be, following the valuation functions of the connectives and quantifiers. Now it is agreed to write $(\mathcal{M}, v) \models_{P a c} \varphi$ : iff $v_{\mathcal{M}}^{\prime} \varphi \in D$, and then $\mathcal{M} \models_{P a c} \varphi$ : iff for any $v,(\mathcal{M}, v) \models_{P a c} \varphi$. Finally, the semantical definition of consequence relation ' $\vDash_{\text {Pac }}$ ' is given by: $\Sigma \vDash_{P a c} \varphi$ : iff for any $\mathcal{L}$-assignment $(\mathcal{M}, v),(\mathcal{M}, v) \models_{P a c}$ $\Sigma \Rightarrow(\mathcal{M}, v) \models_{P a c} \varphi$, where $\Sigma \cup\{\varphi\}$ is a set of $\mathcal{L}$-formulae in Pac.

Thus defined, one can prove that $\vDash_{P a c} \varphi \vee \sim \varphi$, for any formula $\varphi$ in Pac, showing that the law of excluded middle holds for this logic. But on the other hand, the principle of non-contradiction must be given up since for some $\Sigma$ and $\varphi$, it is possible to get $\Sigma \vDash_{P a c} \varphi \wedge \sim \varphi$. Nevertheless, Pac is a paraconsistent logic precisely in the sense that such a contradiction does not entail everything; non-trivial models of some set theories in that logic will provide us with examples of this situation. So let us conclude this concise description with stating expeditiously that, in $P a c$, ' $\wedge$ ', ' $\vee$ ', ' $\rightarrow$ ', ' $\forall$ ', ' $\exists$ ' behave as in classical logic, while the negation ' $\sim$ ' (though acting rather classically on the other primitive connectives and quantifiers) is in some sense weaker than the classical one. This should become less obscure in the light of section 3 .

\footnotetext{
${ }^{2}$ In classical logic, $V=\{0,1\}$ and such a function $R_{\mathcal{M}}$ from $M \times M$ into $V$ becomes identified with a binary relation on $M$, as usual.
} 
REMARK 1. The reader has probably noticed that we have not specified the status of ' $=$ ' yet. As was mentioned, we require ' $=$ ' to be an equality, i.e.

$$
\left\{\begin{array}{cl}
\vDash_{P a c} x=x & \text { (reflexivity) } \\
\{x=y, \varphi\} \vDash_{P a c} \varphi[x \mid y], \text { for any } \mathcal{L} \text {-formula } \varphi \text { in Pac }{ }^{3} & \text { (substitutivity) }
\end{array}\right.
$$

Actually, that can be met by prescribing '=' to satisfy some (finite) set of axioms $I d$ in any $\mathcal{L}$-structure $\mathcal{M}$. This set of axioms will become apparent in the next section.

Finally what follows is the syntactical variant of the ideal calculus that will mainly interest us in this paper:

$$
\left\{\begin{aligned}
E X T & : \equiv \forall \forall y(\forall z(z \in x \Leftrightarrow z \in y) \triangleleft x=y) \\
C O M P & : \equiv\left\{\begin{array}{c}
\text { For any } \mathcal{L} \text {-formula } \varphi \text { in the implicationless fragment of Pac } \\
\exists y \forall x(x \in y \Leftrightarrow \varphi) \quad \text { (y not free in } \varphi \text { ) }
\end{array}\right.
\end{aligned}\right.
$$

Note that the ' $\angle$ ' implication in $E X T$ is actually given by our requirement for ' $=$ ' to be an equality. It is worth remarking that, in these formulations, $E X T$ and $C O M P$ are really compatible in the sense that, for any (suitable) $\mathcal{L}$-formula $\varphi, E X T$ ensures that there is only one set $y$ provided by $C O M P$; in that case, this such set is usually denoted by ' $\{x \mid \varphi\}$ '. In the last section, we will meet a slightly different syntactical variant of the ideal calculus in which this compatibility fails. Then, such a notation ' $\{x \mid \varphi\}$ ' cannot be used unless it is introduced in the language itself to designate a particular set provided by the $\varphi$-instance of the comprehension schema. When each set within a model can be designated in such a way, we shall say that one deals with a term model.

In view of our comprehension schema, only $L P$ formulae can be used for defining a set, and as ' $\rightsquigarrow$ ' does not satisfy modus ponens, we are safe from the set-theoretical version of Curry's paradox. Indeed, we are going to prove that there exist non-trivial models of this calculus or its variants (some of them can contain any classical universe as an initial part). To do that, we will take advantage of a very handy aspect of this logic: the first-order structures can be described classically, as the next section shows.

\footnotetext{
${ }^{3}$ Where $\varphi[x \mid y]$ denotes the $\mathcal{L}$-formula in Pac obtained from $\varphi$ by substituting $y$ for some, but not necessarily all, free occurrences of $x$ (with the proviso that $y$ is free for $x$ in $\varphi)$.
} 
REMARK 2. With regard to the formulation of our syntactical variant of the ideal calculus, one could argue that it would have been more pertinent to consider ' $\Longrightarrow$ ' as primitive implication in the underlying logic. We opted however here for a presentation in which negation is, in some sense, the only "non-classical" primitive connective. Indeed, it is proved in [1] (or in [4]) that the $\{\vee, \wedge, \rightarrow(, \exists, \forall)\}$-fragment of Pac is identical to the corresponding fragment of the two-valued classical logic.

\section{Models in classical setting}

We are going to swap the language of set theory described in the preceding section for the first-order classical calculus built up from $\mathcal{L}^{ \pm}:=\{\in, \notin,=, \neq\}$. The primitive classical connectives and quantifiers will be denoted as usual, namely ' $\neg$ ', ' $\vee$ ', ' $\wedge$ ', ' $\rightarrow$ ', ' $\forall$ ', ' $\exists$ ', so it should be remarked directly that ' $\notin$ ', and ' $\neq$ ' are not abbreviations for the classical negation ' $\neg$ ' of ' $\in$ ' and ' $=$ ' respectively. Basically, in any interpretation, ' $\in$ ' \& ' $\notin$ ' will be weak negation of each other, as well as ' $=$ ' $\&$ ' $\neq$ '. So what we call the $P d$-case is the following:

$$
P d \text {-case }: \equiv(\neg(x \in y) \rightarrow x \notin y) \wedge(\neg(x=y) \rightarrow x \neq y)
$$

REMARK 3. It is interesting to note that the $P d$-case can be restated "positively" in $\mathcal{L}^{ \pm}$, seing that it is equivalent to ' $(x \in y \vee x \notin y) \wedge(x=y \vee x \neq y)$ '. This simple fact will be central in all the constructions we present.

A classical $\mathcal{L}^{ \pm}$-structure satisfying the $P d$-case is called a $P d \mathcal{L}^{ \pm}$-structure. In fact, any $\mathcal{L}$-structure $\mathcal{M}$ for $P a c$ gives rise in a natural way to a $P d \mathcal{L}^{ \pm}$structure $\mathcal{M}^{\Delta}$ on the same universe $M$ by setting :

$$
\text { for any } m, n \in M, \begin{cases}m \epsilon_{\mathcal{M}^{\Delta}} n & : \Leftrightarrow \epsilon_{\mathcal{M}}(m, n) \in\{\imath, 1\} \\ m \notin_{\mathcal{M}^{\Delta}} n & : \Leftrightarrow \epsilon_{\mathcal{M}}^{\prime}(m, n) \in\{0, \imath\} \\ m=_{\mathcal{M}^{\Delta}} n & \Leftrightarrow \Leftrightarrow=_{\mathcal{M}}^{\prime}(m, n) \in\{\imath, 1\} \\ m \neq_{\mathcal{M}^{\Delta}} n & : \Leftrightarrow=_{\mathcal{M}}^{\prime}(m, n) \in\{0, \imath\}\end{cases}
$$

And conversely, from any $\operatorname{Pd} \mathcal{L}^{ \pm}$-structure $\mathcal{M}$, one can define an $\mathcal{L}$-structure $\mathcal{M}^{\nabla}$ for $P a c$, on the same universe $M$, as follows:

$$
\text { for any } m, n \in M,\left\{\begin{array}{lllll}
\epsilon_{\mathcal{M} \nabla^{\prime}}(m, n)=1 & : \Leftrightarrow & m \epsilon_{\mathcal{M}} n & \wedge & \neg\left(m \notin_{\mathcal{M}} n\right) \\
\epsilon_{\mathcal{M} \nabla^{\prime}}(m, n)=\imath & : \Leftrightarrow & m \epsilon_{\mathcal{M}} n & \wedge & m \notin_{\mathcal{M}} n \\
\epsilon_{\mathcal{M} \nabla^{\prime}}(m, n)=0 & : \Leftrightarrow & \neg\left(m \epsilon_{\mathcal{M}} n\right) & \wedge & m \notin_{\mathcal{M}} n \\
=_{\mathcal{M}^{\nabla}}(m, n)=1 & : \Leftrightarrow & m=_{\mathcal{M}} n & \wedge & \neg\left(m \neq_{\mathcal{M}} n\right) \\
=_{\mathcal{M}} \nabla^{\prime}(m, n)=\imath & : \Leftrightarrow & m=_{\mathcal{M}} n & \wedge & m \neq_{\mathcal{M}} n \\
=_{\mathcal{M}} \nabla^{\prime}(m, n)=0 & : \Leftrightarrow & \neg\left(m=_{\mathcal{M}} n\right) & \wedge & m \neq_{\mathcal{M}} n
\end{array}\right.
$$

In this way, $\mathcal{M}^{\Delta \nabla}=\mathcal{M}$, for any $\mathcal{L}$-structure $\mathcal{M}$ for Pac, and $\mathcal{M}^{\nabla \Delta}=\mathcal{M}$, for any $P d \mathcal{L}^{ \pm}$-structure $\mathcal{M}$. It remains now to describe the translation at the 
language level. In view of our semantical translation above, any $\mathcal{L}$-formula $\varphi$ in Pac gives rise actually to a pair $\left(\varphi^{+}, \varphi^{-}\right)$of $\mathcal{L}^{ \pm}$-formulae which are defined inductively as follows:

$$
\begin{aligned}
& \begin{array}{l|l|l}
(x \in y)^{+} \quad \text { is } \quad \text { ' } x \in y^{\prime} & (x \in y)^{-} \quad \text { is } \quad \text { ' } x \notin y \text { ' }
\end{array}
\end{aligned}
$$

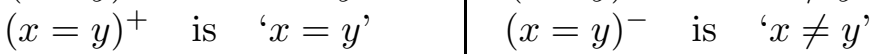

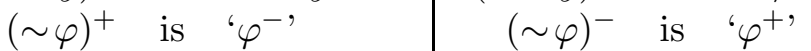

$$
\begin{aligned}
& (\varphi \wedge \psi)^{+} \quad \text { is ' } \varphi^{+} \wedge \psi^{+}, \quad(\varphi \wedge \psi)^{-} \quad \text { is ' } \varphi^{-} \vee \psi^{-} \text {, } \\
& (\varphi \vee \psi)^{+} \quad \text { is ' } \varphi^{+} \vee \psi^{+}, \quad(\varphi \vee \psi)^{-} \quad \text { is ' } \varphi^{-} \wedge \psi^{-} \text {' } \\
& (\varphi \rightarrow \psi)^{+} \quad \text { is ' } \varphi^{+} \rightarrow \psi^{+}, \quad(\varphi \rightarrow \psi)^{-} \quad \text { is ' } \varphi^{+} \wedge \psi^{-} \text {' } \\
& (\forall x \varphi)^{+} \text {is ' } \forall x \varphi^{+}, \quad(\forall x \varphi)^{-} \text {is ' } \exists x \varphi^{-} \text {' } \\
& (\exists x \varphi)^{+} \text {is ' } \exists x \varphi^{+}, \quad(\exists x \varphi)^{-} \quad \text { is ' } \forall x \varphi^{-} \text {' }
\end{aligned}
$$

Indeed, in this way, one can prove that, for any $\mathcal{L}$-assignment $(\mathcal{M}, v)$ for Pac, we have:

$$
(\mathcal{M}, v) \models_{P a c} \varphi \Leftrightarrow\left(\mathcal{M}^{\Delta}, v\right) \models_{C l a} \varphi^{+} \&(\mathcal{M}, v) \models_{P a c} \sim \varphi \Leftrightarrow\left(\mathcal{M}^{\Delta}, v\right) \models_{C l a} \varphi^{-}
$$

and therefrom, for any $\mathcal{L}^{ \pm}$-assignment $(\mathcal{M}, v)$ :

$$
\left(\mathcal{M}^{\nabla}, v\right) \models_{P a c} \varphi \Leftrightarrow(\mathcal{M}, v) \models_{C l a} \varphi^{+} \&\left(\mathcal{M}^{\nabla}, v\right) \models_{P a c} \sim \varphi \Leftrightarrow(\mathcal{M}, v) \models_{C l a} \varphi^{-}
$$

Thus we immediately get:

$$
\Sigma \vDash_{P a c} \varphi \Leftrightarrow \Sigma^{+} \cup\left\{{ }^{\prime} P d \text {-Case' }\right\} \vDash_{C l a} \varphi^{+}
$$

(where $\Sigma^{+}:=\left\{\psi^{+} \mid \psi \in \Sigma\right\}$.)

REMARK 4. Let us just mention that this is actually the way some paraconsistent set theories were presented in [11], but without specifying any references to Pac (nor its synonyms).

Remark 5. With regard to Remark 1 , it is now fairly easy to find a (finite) set of axioms $I d$ to be satisfied in any $\mathcal{L}$-structure $\mathcal{M}$ for Pac so that '=' be an equality. Indeed, let $C g$ be the (finite) set of axioms for ' $=$ ' ensuring that a $\mathcal{L}^{ \pm}$-congruence, i.e.:

$$
C g: \equiv\left\{\begin{array}{l}
\bullet \text { the axioms for ' }=\text { ' to be an equivalence relation; } \\
\bullet \text { for any } R \in \mathcal{L}^{ \pm} \backslash\{=\}, \\
\quad \forall x \forall y(x=y \rightarrow(\forall z(z R x \leftrightarrow z R y) \wedge \forall z(x R z \leftrightarrow y R z))) .
\end{array}\right.
$$

and then consider $I d$ in $P a c$ such that $\mathrm{Id}^{+}=\mathrm{Cg}^{4}$

\footnotetext{
be:

${ }^{4}$ Incidentally, a suitable way of defining a notion of congruence for ' $=$ ' in Pac could

$$
\left\{\begin{array}{l}
\text { for any } R \in \mathcal{L}, \\
\forall x \forall y(x=y \rightarrow(\forall z(z R x \Leftrightarrow z R y) \wedge \forall z(x R z \Leftrightarrow y R z))) .
\end{array}\right.
$$
}


It is worth observing that, for any $\mathcal{L}$-formula $\varphi$ in $P a c, \varphi^{+}$and $\varphi^{-}$are pseudo-positive in $\mathcal{L}^{ \pm} .{ }^{5}$ Clearly, the translation described above consists in "absorbing" the non-classical (weak) negation ' $\sim$ ' of Pac in $\mathcal{L}^{ \pm}$. Now we bring it out again by officially defining the weak negation $\bar{\varphi}$ of any pseudopositive $\mathcal{L}^{ \pm}$-formula $\varphi$ inductively as follows:

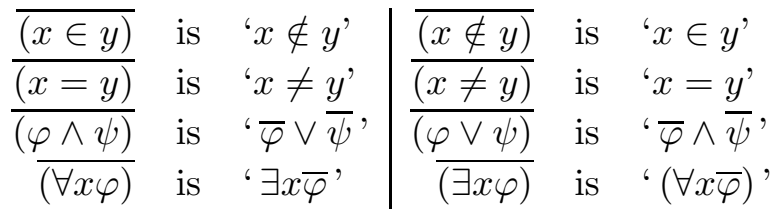

$$
\begin{aligned}
& \overline{(\varphi \rightarrow \psi)} \text { is } \varphi \wedge \bar{\psi}
\end{aligned}
$$

Actually, the $P d$-case entails (classically) $\varphi \vee \bar{\varphi}$, for any pseudo-positive $\mathcal{L}^{ \pm}$-formula $\varphi$, and it is worth noting that the weak negation of any pseudopositive $\mathcal{L}^{ \pm}$-formula is always positive; in fact, $\varphi$ is positive if and only if $\overline{\bar{\varphi}}=$ $\varphi$. The analogue in $P a c$ to such a formula is within $L P$, the implicationless fragment of $P a c$. So, by virtue of our formulation of the ideal calculus, these formulae will play a central role in the construction of its models. Here is precisely that so-called ideal calculus translated into $\mathcal{L}^{ \pm}$:

$$
\left\{\begin{aligned}
E X T & : \equiv \forall \forall y(\forall z((z \in x \leftrightarrow z \in y) \wedge(z \notin x \leftrightarrow z \notin y)) \leftrightarrow x=y) \\
C O M P & : \equiv\left\{\begin{array}{l}
\text { For any positive } \mathcal{L}^{ \pm} \text {-formula } \varphi, \\
\exists y \forall x((x \in y \leftrightarrow \varphi) \wedge(x \notin y \leftrightarrow \bar{\varphi})) \text { (y not free in } \varphi)
\end{array}\right.
\end{aligned}\right.
$$

Basically, the techniques that will be used to construct models of this calculus are essentially based upon a well-known property of positive formulae stating that such formulae are preserved under surjections:

Theorem 1 (Projective Lemma).

Let $\mathcal{M}, \mathcal{N}$ be any $\mathcal{L}^{ \pm}$-structures and $f: \mathcal{M} \rightarrow \mathcal{N}$ a surjective $\mathcal{L}^{ \pm}$-morphism. Then, for any positive $\mathcal{L}^{ \pm}$-formula $\varphi\left(x_{1}, \ldots, x_{k}\right)$ and any $m_{1}, \ldots, m_{k} \in M$, we have $\mathcal{M} \models \varphi\left[m_{1}, \ldots, m_{k}\right] \Rightarrow \mathcal{N} \models \varphi\left[f^{\prime} m_{1}, \ldots, f^{\prime} m_{k}\right]$

Proof. This can be easily checked by induction on the complexity of $\varphi$. Note that the surjectivity of $f$ is required for the universal quantifier case only.

\footnotetext{
${ }^{5}$ Where a $\mathcal{L}^{ \pm}$-formula is said to be pseudo-positive if it can be constructed from atomic $\mathcal{L}^{ \pm}$-formulae without using ' $\neg$ ', and it is said to be positive if it can be constructed without using ' $\neg$ ' nor ' $\rightarrow$ '. This terminology follows the one used to in classical logic.
} 
We will see that a larger class of formulae has also an exemplary behavior with respect to some specific operations involved in our constructions; so let us conclude this section with defining inductively the class of bounded positive (BP) $\mathcal{L}^{ \pm}$-formulae:

1. Any atomic $\mathcal{L}^{ \pm}$-formula is BP.

2. If $x$ is a variable and if $\varphi, \psi$ are $\mathrm{BP}$, so are ' $\varphi \wedge \psi$ ', ' $\varphi \vee \psi$ ', ' $\forall x \varphi^{\prime}$, ' $\exists x \varphi^{\prime}$.

3. If $x, y$ are distinct variables and if $\varphi$ is $\mathrm{BP}$, so are ' $\forall x(x \in y \rightarrow \varphi)$ ' and ' $\forall x(x \notin y \rightarrow \varphi)$ '.

Particularly, the positive formulae are those obtained without using rule 3 , and so, some strong negations ' $\neg$ ' are allowed in BP formulae through the implications ' $x \in y \rightarrow \cdots$ ' and ' $x \notin y \rightarrow \cdots$ '. Since any BP formula is pseudo-positive, this notion has an analogue in Pac.

\section{About extensionality}

Here we are going to present a result providing a way of recovering extensionality within an $\mathcal{L}^{ \pm}$-structure that initially fails to satisfy it. This will be used in the last section of the paper. It turns out that our formulation of extentionality lends easily itself to an adaptation of the bisimulation technique required in classical analogous circumstances. But, indeed, the key point ensuring that this adaptation really works out is the positiveness of the Pd-case.

\section{Extensions and p-sets}

In any $\mathcal{L}^{ \pm}$-structure $\mathcal{M} \equiv\left\langle M ; \mathcal{L}_{\mathcal{M}}^{ \pm}\right\rangle$, we define the extension and the antiextension of each $x \in M$ respectively as follows:

$$
[x]_{\mathcal{M}}^{+}:=\left\{y \mid y \epsilon_{\mathcal{M}} x\right\} \quad \& \quad[x]_{\mathcal{M}}^{-}:=\left\{y \mid y \notin_{\mathcal{M}} x\right\}
$$

So the ' $\in$ ' part of the $P d$-case means exactly that $[x]_{\mathcal{M}}^{+} \cup[x]_{\mathcal{M}}^{-}=M$, for any $x \in M$. Naturally, an $\mathcal{M}$-set $x$ shall be said to be classical if $[x]_{\mathcal{M}}^{+} \cap[x]_{\mathcal{M}}^{-}=\emptyset$. In that setting, extensionality can be rephrased just like this:

$$
E X T \equiv \forall x \forall y\left(\left([x]^{+}=[y]^{+} \wedge[x]^{-}=[y]^{-}\right) \rightarrow x=y\right)
$$

Therefore, in any $P d \mathcal{L}^{ \pm}$-structure $\mathcal{M}$ satisfying $E X T$, an $\mathcal{M}$-set can be identified with a covering pair $(A, B)$ of subsets of $M$. Such a pair is called a paraconsistent set, or $p$-set, over $M$ (as in [6]). 


\section{A bisimulation technique}

Now let $E q(M)$ denote the complete lattice of equivalence relations on $M$. If $R$ is any binary relation on $M$ and if $\sim \in E q(M)$, then one can define naturally a new binary relation $\widetilde{R}$ on $M$ by modulating $R$ with $\sim$, i.e.

$$
x \widetilde{R} y: \Leftrightarrow \exists x^{\prime} \sim x, \exists y^{\prime} \sim y: x^{\prime} R y^{\prime} .
$$

Thus, starting with $\mathcal{M}$ and $\sim \in E q(M)$, one can define a new $\mathcal{L}^{ \pm}$-structure $\widetilde{\mathcal{M}}$ on the same universe by modulating each relation $R_{\mathcal{M}}$ for $R \in \mathcal{L}_{\mathcal{M}}^{ \pm}$in this way. Obviously, if $\sim$ is $=_{\mathcal{M}}$, then $\widetilde{\mathcal{M}}$ is nothing but $\mathcal{M}$ (since $=_{\mathcal{M}}$ is a congruence). However, it should be noted that $\widetilde{\subseteq}_{\mathcal{M}}$ is not in general an equivalence on $M$ (transitivity may fail). Anyway, if $=_{\mathcal{M}}$ is the real equality on $M$ (i.e. $\mathcal{M}$ is normal), then $\cong_{\mathcal{M}}$ is $\sim$ and it is actually a congruence on $M$ for $\widetilde{\in}_{\mathcal{M}}, \widetilde{\notin}_{\mathcal{M}}$ and $\widetilde{F}_{\mathcal{M}}$, as the definition of these clearly shows. Moreover, if $\mathcal{M}$ is a $\operatorname{Pd} \mathcal{L}^{ \pm}$-structure, so is $\widetilde{\mathcal{M}}$. This follows from the following observation: for any $R \in \mathcal{L}^{ \pm}, R_{\mathcal{M}} \subseteq \widetilde{R}_{\mathcal{M}}=: R_{\widetilde{\mathcal{M}}}$. Hence, the identity map on $M$ is a surjective morphism from $\mathcal{M}$ onto $\widetilde{\mathcal{M}}$, and by the projective lemma, any positive formula is preserved, so is the $P d$-case.

We now move towards introducing the concept of bisimulation in this context.

Definition. $\sim \in E q(M)$ is a bisimulation (in $\mathcal{M}$ ) if the notions of extensions are preserved, i.e. for any $x \in M,[x]_{\widetilde{\mathcal{M}}}^{+}=\left([x]_{\mathcal{M}}^{+}\right)^{\sim}$ and $[x]_{\widetilde{\mathcal{M}}}^{-}=\left([x]_{\mathcal{M}}^{-}\right)^{\sim}$, where $(A)^{\sim}:=\{x \in M \mid \exists a \in A: a \sim x\}$, for any $A \subseteq M$.

It is interesting to note that even bounded positive formulae are preserved when one deals with a bisimulation. This will turn out to be essential in the last section and here is the key to the proof :

Proof. Assume that $\varphi(x, y, z, \ldots)$ is preserved and let us check that so is ' $\forall x(x \in y \rightarrow \varphi(x, y, z, \ldots))$ '. Suppose therefore that $\mathcal{M} \models \forall x(x \in y \rightarrow$ $\varphi(x, y, z, \ldots))$ and, then, let $m, n, p \ldots \in M$ with $m \in_{\widetilde{\mathcal{M}}} n$. Since $\sim$ is a bisimulation, we know there exists $m^{\prime} \in M$, such that $m^{\prime} \sim m$ and $m^{\prime} \in_{\mathcal{M}} n$. So, by hypothesis, we have $\mathcal{M} \models \varphi\left[m^{\prime}, n, p, \ldots\right]$. Now, being assumed that $\varphi$ is preserved, we have $\widetilde{\mathcal{M}} \models \varphi\left[m^{\prime}, n, p, \ldots\right]$ and, as $m^{\prime} \sim m$, this yields $\widetilde{\mathcal{M}} \models \varphi[m, n, p, \ldots]$, showing that $\widetilde{\mathcal{M}} \models \forall x(x \in y \rightarrow \varphi(x, y, z, \ldots))$. A similar argument goes with $\notin$ instead of $\in$.

The concept of bisimulation can be handled more easily by defining the following monotone operator $(\cdot)^{+}$on $E q(M)$ :

$$
x \sim^{+} y \quad: \Leftrightarrow \quad\left([x]_{\mathcal{M}}^{+}\right)^{\sim}=\left([y]_{\mathcal{M}}^{+}\right)^{\sim} \quad \& \quad\left([y]_{\mathcal{M}}^{-}\right)^{\sim}=\left([y]_{\mathcal{M}}^{-}\right)^{\sim}
$$


Thus, one can prove that $\sim$ is a bisimulation if and only if $\sim \subseteq \sim^{+}$and then, for a bisimulation, we have $\sim=\sim^{+}$if and only if $\widetilde{\mathcal{M}} \models E X \bar{T}$. Now it becomes easy to prove the aimed result of this section:

Theorem 2.

For any normal $\mathcal{L}^{ \pm}$-structure $\mathcal{M}$, there exists a finest bisimulation $\sim$ on $M$ such that $\widetilde{\mathcal{M}}=E X T$.

Proof. ' $\sim$ ' is simply the least fixed point of the $(\cdot)^{+}$operator. It can be obtained inductively by iterating this operator from $=_{\mathcal{M}}$ (which is supposed to be the real equality on $M$ ):

$$
\left\{\begin{aligned}
\sim_{0} & :==_{\mathcal{M}} \\
\sim_{\alpha+1} & :=\sim_{\alpha}^{+} \\
\sim_{\lambda} & :=\bigcup_{\alpha<\lambda} \sim_{\alpha}(\lambda \text { limit })
\end{aligned}\right.
$$

As we start with $=_{\mathcal{M}}$, which is obviously a bisimulation, and since $(\cdot)^{+}$is monotone, each $\sim_{\alpha}$ is actually a bisimulation. Then, the ' $\sim$ ' we are looking for is $\sim_{\alpha_{0}}$ where $\alpha_{0}$ is the least ordinal $\alpha$ such that $\sim_{\alpha+1}=\sim_{\alpha}$.

\section{Topological models, "Hyper-Frege" and the axiom of choice}

The models we describe in this section first appeared in [11]. The technique that was used for their construction is actually an adaptation of the one used to build extensional models of positive comprehension (see [9]). We shall briefly survey that construction exhibiting by the way some properties of these models which will give rise to a very special paraconsistent set theory called "Hyper-Frege". The status of the axiom of choice in that theory will be discussed.

\section{A topological model}

Many proofs are omitted but can be found in [11].

We start with any non-empty finite set $X$ and we define a sequence $\left(X_{n} \mid n \in \omega\right)$ from $X$ by induction:

$$
\left\{\begin{array}{l}
X_{0}:=X \\
X_{n+1}:=\left\{(\zeta, \xi) \mid \zeta \cup \xi=X_{n}\right\} \quad(n \in \omega)
\end{array}\right.
$$

Now let $s$ be any surjection from $X_{1}$ onto $X_{0}$. One can naturally extend $s$ to the higher levels inductively by setting $s^{\prime}(\zeta, \xi):=\left(s^{\prime \prime} \zeta, s^{\prime \prime} \xi\right)$, and then, 
set up each level $X_{n}$ as a $\mathcal{L}^{ \pm}$-structure in such a way that $s$ becomes an epimorphism between two consecutive levels. To do that, we proceed as follows:

-) $\epsilon_{\mathcal{X}_{0}}=\notin_{\mathcal{X}_{0}}:=X^{2}$ and, for all $n \in \omega$, we define ' $\in$ ' and ' $\notin$ ' in $X_{n+1}$ by means of their extensions like this:

$$
[(\zeta, \xi)]_{\mathcal{X}_{n+1}}^{+}:=s^{-1 "} \zeta \quad \& \quad[(\zeta, \xi)]_{\mathcal{X}_{n+1}}^{-}:=s^{-1 "} \xi \quad ;
$$

-) for all $n \in \omega,=_{\mathcal{X}_{n}}$ is the real equality on $X_{n}$ and ' $\neq$ ' is defined by the (positive) formula $\delta(x, y): \equiv \exists z((z \in x \wedge z \notin y) \vee(z \notin x \wedge z \in y))$.

Furthermore, it can be easily checked that, in this way, for each $n \in \omega, \mathcal{X}_{n}$ is a $P d \mathcal{L}^{ \pm}$-structure satisfying $E X T^{\sharp}$ which is defined by :

$$
E X T^{\sharp}: \equiv E X T \wedge \forall x \forall y(x \neq y \leftrightarrow \delta(x, y))
$$

REMARK 6. We should emphasize that this is actually a stronger version of extensionality, which corresponds in Pac to:

$$
E X T^{\sharp} \equiv \forall x \forall y(\forall z(z \in x \Leftrightarrow z \in y) \Longrightarrow x=y)
$$

Notice also that it gives us a nice characterization of ' $\neq$ ' seeing that:

$$
x \neq x \Leftrightarrow \delta(x, x) \Leftrightarrow[x]^{+} \cap[x]^{-} \neq \emptyset \Leftrightarrow x \text { is paradoxical }
$$

Now the $\mathcal{L}^{ \pm}$-structure we are interested in will be the projective limit of those we have just defined:

$\mathcal{X}_{\omega}: \equiv \lim _{n \in \omega} \mathcal{X}_{n}$, i.e. $\left\{\begin{array}{l}X_{\omega}:=\left\{x=\left(x_{n} \mid n \in \omega\right) \in \prod_{n \in \omega} X_{n} \mid \forall n \in \omega, s^{\prime} x_{n+1}=x_{n}\right\} \\ \text { For any } R \in \mathcal{L}^{ \pm}, \quad x R_{\mathcal{X}_{\omega}} y: \Leftrightarrow \forall n \in \omega, x_{n} R_{\mathcal{X}_{n}} y_{n}\end{array}\right.$

Then, one can prove that $\mathcal{X}_{\omega}$ is still a $P d \mathcal{L}^{ \pm}$-structure satisfying $E X T^{\sharp}$, and especially for this, a compactness argument is required. The proof can be found in [11], but we shall briefly recall here which topology is involved before looking further into the properties of the model.

We simply equip $X_{\omega}$ with the natural topology induced by the product topology when each $X_{n}$ is fitted with the discrete one (by the way, we would remind the reader that any $X_{n}$ is finite). Thus, it can be easily shown that $X_{\omega}$ is compact and that a basis for its topology is given by the clopen sets $U_{\zeta}:=\left\{x \in X_{\omega} \mid x_{n}=\zeta\right\}, n \in \omega, \zeta \in X_{n}$. Notice that $U_{\zeta} \subseteq U_{\zeta^{\prime}}$ iff there exists $k \in \omega$ such that $s^{k} \zeta=\zeta^{\prime}$; moreover, if $U_{\zeta} \nsubseteq U_{\zeta^{\prime}}$ and $U_{\zeta} \nsupseteq U_{\zeta^{\prime}}$, then $U_{\zeta} \cap U_{\zeta^{\prime}}=\emptyset$. 
This topology really fits in with our $\mathcal{L}^{ \pm}$-structure as one can prove that the interpretations of the primitive symbols in $X_{\omega}$ are "continuous" with regard to that topology. More precisely, let us say that an $\mathcal{L}^{ \pm}$-formula $\varphi\left(x_{0}, \ldots, x_{n}\right)$ is continuous in $X_{\omega}$ if $\left\{\left(x_{0}, \ldots, x_{n}\right) \mid \mathcal{X}_{\omega} \models \varphi\left[x_{0}, \ldots, x_{n}\right]\right\}$ is closed in $X_{\omega}^{n}$. Then, it is proved in [11] that actually any BP $\mathcal{L}^{ \pm}$-formula is continuous in $X_{\omega}$. Particularly, for any $y \in X_{\omega},\left([y]_{\mathcal{X}_{\omega}}^{+},[y]_{\mathcal{X}_{\omega}}^{-}\right)$is a covering pair of closed subsets of $X_{\omega}$. The main property of the model is in a sense the converse:

for any covering pair $(A, B)$ of closed subsets of $X_{\omega}$, there exists an $\mathcal{X}_{\omega}$-set $y$ such that $[y]_{\mathcal{X}_{\omega}}^{+}=A$ and $[y]_{\mathcal{X}_{\omega}}^{-}=B$.

(Again, for a proof see [11].)

Now let $(\varphi, \psi)$ be a pair of BP $\mathcal{L}^{ \pm}$-formulae such that $\mathcal{X}_{\omega} \models \varphi \vee \psi$. By virtue of the continuity of these formulae, the property above tells us that there exists a (unique) set $y$ such that

$$
[y]_{\mathcal{X}_{\omega}}^{+}=\left\{x \in X_{\omega} \mid \mathcal{X}_{\omega} \models \varphi\right\} \quad \& \quad[y]_{\mathcal{X}_{\omega}}^{-}=\left\{x \in X_{\omega} \mid \mathcal{X}_{\omega} \models \psi\right\}
$$

This set shall be denoted by ' $\{x \mid \varphi\{\psi\}$ '. In particular, when $\varphi$ is positive and $\psi$ is exactly $\bar{\varphi}$, we get ' $\{x \mid \varphi\}$ ' showing that $\mathcal{X}_{\omega} \models C O M P$.

In order to formulate another related property in a more expressive way, we need the following definition:

Definition. $x$ is less paradoxical than $y$ when $[x]^{+} \subseteq[y]^{+}$and $[x]^{-} \subseteq[y]^{-}$.

In these terms, a straightforward consequence of the main property mentioned above can be put into words as follows:

for every covering pair $(A, B)$ of subsets of $X_{\omega}$, there exists a minimal paradoxical $\mathcal{X}_{\omega}$-set $y$ such that $A \subseteq[y]_{\mathcal{X}_{\omega}}^{+}$and $B \subseteq[y]_{\mathcal{X}_{\omega}}^{-}$.

This shows that the underlying topological structure on $X_{\omega}$ really confers a very characteristic property on the model that is axiomatized next.

\section{"Hyper-Frege"}

As was seen, each set in the model can be identified with a covering pair of closed subsets of the universe, which actually constitutes a subalgebra of the "paraconsistent boolean algebra" of p-sets over $X_{\omega}$ (see [6]). For instance, the underlying order of this algebra corresponds to the most natural 
interpretation of "inclusion" in the model, according to our formulation of extensionality in Pac, which is the following:

$$
\begin{aligned}
x \sqsubseteq y & : \Leftrightarrow \forall z(z \in x \Longrightarrow z \in y) \\
& \Leftrightarrow[x]^{+} \subseteq[y]^{+} \&[y]^{-} \subseteq[x]^{-}
\end{aligned}
$$

The bottom element is the "empty set" $\Lambda$ and the top element is the "universal set" $\mathrm{V}$, which are defined in our model by:

$$
\begin{array}{ll}
{[\Lambda]_{\mathcal{X}_{\omega}}^{+}:=\emptyset} & {[\mathrm{V}]_{\mathcal{X}_{\omega}}^{+}:=X_{\omega}} \\
{[\Lambda]_{\mathcal{X}_{\omega}}^{-}:=X_{\omega}} & {[\mathrm{V}]_{\mathcal{X}_{\omega}}^{-}:=\emptyset}
\end{array}
$$

These sets are classical. Note incidentally that, within the algebra of $p$-sets over $X_{\omega}$, the class of classical sets of the model can be identified with the boolean algebra of clopen subsets of $X_{\omega}$. Furthermore, it can be shown that the class of hereditarily classical well-founded sets in $X_{\omega}$ yields a model for $Z F$ except the axiom of infinity (see below). Beside these classical sets, our model is replete with paraconsistent ones, such as the Russell set $R:=\{x \mid$ $x \notin x\}$, so that it might frame an elegant domain of investigation of such sets.

Anyway, we shall not elaborate on the properties of the model here but content ourselves with presenting a general first order theory in $\mathcal{L}^{ \pm}$which arises naturally from those properties pointed out so far. This theory will be called "Hyper-Frege" HF. It can be axiomatized as follows:

$H F: \equiv\left\{\begin{array}{l}\bullet \text { First-order predicate calculus with identity ' }=\text { ' in } \mathcal{L}^{ \pm} \\ \bullet \text { The } P \text { d-case } \\ \bullet \text { EXT } \\ \bullet \text { Closure Schema: } \\ \quad \text { for every pair }(\varphi, \psi) \text { of } \mathcal{L}^{ \pm} \text {-formulae, the following axiom: } \\ \forall x(\varphi \vee \psi) \rightarrow \text { "there exists a minimal paradoxical set } y \text { such that } \\ \quad \forall x(\varphi \rightarrow x \in y) \wedge \forall x(\psi \rightarrow x \notin y) \text { " }\end{array}\right.$

- Bicomprehension Schema: for every pair $(\varphi, \psi)$ of bounded positive $\mathcal{L}^{ \pm}$-formulae:

$$
\forall x(\varphi \vee \psi) \rightarrow \exists y(\forall x(x \in y \leftrightarrow \varphi) \wedge \forall x(x \notin y \leftrightarrow \psi)
$$

This theory appears in a sense to be the natural paraconsistent counterpart of the positive set theory $G P K^{+}$, introduced and deeply investigated by Esser in [7]. He showed that $G P K_{\infty}^{+}$interprets $Z F$, where $G P K_{\infty}^{+}: \equiv G P K^{+}+$"there exists an infinite (von Neumann's) ordinal". At present, our research consists in finding a relevant formulation in $H F$ of an 
axiom of infinity $I$ that would permit to show that $H F_{\infty}: \equiv H F+I$ interprets $G P K_{\infty}^{+}$, and therefrom $Z F$. Moreover, we conjecture that $H F_{\infty}$ should be "modelizable" in $Z F$ plus some reasonable large cardinal assumption.

REMARK 7. By the way, let us draw the reader's attention to the fact that, though $X_{\omega}$ is infinite, $\mathcal{X}_{\omega}$ does not satisfy any relevant axiom of infinity that would yield transfinite induction. For instance, $\mathcal{X}_{\omega} \models \exists x(\Lambda \in x \wedge \forall y(y \in$ $x \rightarrow y \cup\{y\} \in x)$ ). Indeed, $\mathrm{V}$ is such an ' $x$ ', whatever ' $\cup$ ' and ' $\{\cdot\}$ ' might mean in $\mathcal{X}_{\omega}$. Although in $Z F$ this axioms provides the existence of the least infinite ordinal $\omega$, nothing like that happens here.

\section{The axiom of choice in $H F$}

Esser proved in [8] that $G P K_{\infty}^{+} \vdash \neg A C$, no matter how one formulates the axiom of choice $(A C)$. In view of what has been argued, it is reasonable to conjecture that the situation is similar in $H F_{\infty}$. However, $H F+A C$ is consistent. Indeed, we can prove that $\mathcal{X}_{\omega} \models$ "Every set can be well-ordered". It suffices in fact to show that $\mathrm{V}$ can be. To see this, we begin with defining a "continuous" ordering on $X_{\omega}$. Here is the procedure:

-) Choose any linear ordering $\leqslant_{n}$ on each $X_{n}$ in such a way that $s$ is order preserving. This can be done by choosing an ordering $\leqslant_{n+1}$ satisfying the following condition:

$$
\forall \zeta, \xi \in X_{n}\left(\zeta<_{n} \xi \Rightarrow \forall \varsigma \in s^{-1 "}\{\zeta\}, \forall \varrho \in s^{-1 "}\{\xi \xi\}, \varsigma<_{n+1} \varrho\right)
$$

-) Define $\leqslant_{\omega}$ as the restriction of the lexical product of the $\leqslant_{n}$ 's on $X_{\omega}$, i.e.

$$
x<_{\omega} y \Leftrightarrow x_{n_{0}}<_{n_{0}} y_{n_{0}} \text {, where } n_{0} \text { is the least } n \in \omega \text { such that } x_{n} \neq y_{n}
$$

Since $s$ is order preserving, that actually amounts to ' $\exists n \in \omega: x_{n}<_{n} y_{n}$ ', and it is now very easy to show that $\leqslant_{\omega}$ is "continuous":

Proof. This amounts to proving that $U:=\left\{(x, y) \mid x<_{\omega} y\right\}$ is open in $X_{\omega}^{2}$. So let $a<_{\omega} b$. Then, there exists $n \in \omega$ such that $a_{n}<_{n} b_{n}$, and we have $U_{a_{n}} \times U_{b_{n}} \subseteq U$.

Basically, the continuity of this ordering ensures that it can be coded in $\mathcal{X}_{\omega}$, in the sense that there exists $r \in X_{\omega}$ such that:

$$
(x, y) \in_{\mathcal{X}_{\omega}} r \Leftrightarrow x \leqslant_{\omega} y
$$


This requires some further explanations:

We have first to specify the meaning of an expression such as ' $(x, y) \in r$ ' in $\mathcal{X}_{\omega}$. Of course, this would mean that ' $r$ ' is a set of ordered pairs and that ' $(x, y)$ ' is one of them. So we need to define a notion of ordered pair in the model or more generally in the theory. This can be done naturally by using the usual Kuratowski's definition, i.e. ' $(x, y):=\{\{x\},\{x, y\}\}$ ', provided we have fixed a notion of pair in $H F$. The one we choose here is defined by:

$$
\{a, b\}_{L}:=\{x \mid x=a \vee x=b \prec x=x\}
$$

This definition might seem quite disconcerting as for any $x, x \notin\{a, b\}_{L}$ ! Anyhow, it ensures that a formula such as ' $z=\{x, y\}_{L}$ ' is BP. Indeed, in $H F$, this formula is equivalent to:

$$
\forall u(u \in z \rightarrow(u=x \vee u=y)) \wedge(x \in z \wedge y \in z) \wedge \forall u(u \notin z)
$$

Now the reader should easily convince himself that a formula such as ' $z=$ $(x, y)_{L}{ }^{\prime}$ is $\mathrm{BP}$ as well. Thus, in view of the continuity of $\leqslant_{\omega}$, the formula ' $\exists x \exists y\left(z=(x, y)_{L} \wedge x \leqslant_{\omega} y\right)^{\prime}$ ' should be continuous in $X_{\omega}$. Hence, there does exist $r \in X_{\omega}$ such that:

$$
\left\{\begin{array}{l}
{[r]_{\mathcal{X}_{\omega}}^{+}:=\left\{z \in X_{\omega} \mid \mathcal{X}_{\omega} \models \exists x \exists y\left(z=(x, y)_{L} \wedge x \leqslant_{\omega} y\right)\right\}} \\
{[r]_{\mathcal{X}_{\omega}}^{-}:=X_{\omega}}
\end{array}\right.
$$

And so we have $\mathcal{X}_{\omega} \models(x, y)_{L} \in r \leftrightarrow x \leqslant_{\omega} y$.

Now it remains to show that $\leqslant_{\omega}$ is really a "well-ordering" on $\mathrm{V}$ in $\mathcal{X}_{\omega}$. The compactness of $X_{\omega}$ will be decisive once more:

Proof. We are going to show that

$$
\mathcal{X}_{\omega} \models \forall y\left(\Lambda \neq y \sqsubseteq \mathrm{V} \rightarrow \exists a\left(a \in y \wedge \forall x\left(x \in y \rightarrow a \leqslant_{\omega} x\right)\right)\right) .
$$

So let $\Lambda \neq y \sqsubseteq \mathrm{V}$. Then $[y]_{\mathcal{X}_{\omega}}^{+}$is non-empty and we can define $a:=\left(a_{n} \mid n \in \omega\right)$ by setting $a_{n}:=\min _{\aleph_{n}}\left\{x_{n} \mid x \in_{\mathcal{X}_{\omega}} y\right\}$. Now, as $[y]_{\mathcal{X}_{\omega}}^{+}$is closed in $X_{\omega}$, so is $A_{n}:=U_{a_{n}} \cap[y]_{\mathcal{X}_{\omega}}^{+}$, for all $n \in \omega$. Since $s$ is order preserving, it is also easy to see that, for all $n \in \omega, \bigcap\left\{A_{k} \mid k \leqslant n\right\}=A_{n}$ and then is non-empty. Thus, so is $\bigcap\left\{A_{k} \mid k \leqslant \omega\right\}$ by compactness of $X_{\omega}$, and as this intersection is nothing but $\{a\}$, we get $a \in_{\mathcal{X}_{\omega}} y$. On the other hand, by definition, it is clear that $a \leqslant_{\omega} x$ for all $x \in_{\mathcal{X}_{\omega}} y$. 


\section{Extending any classical universe to a paraconsistent one}

Here we prove that any classical universe of set theory (supposed to be extensional) can be extended to a paraconsistent one by using a term model construction. The inductive technique used here is the "dual" adaptation of the one introduced in [10], where the author produced a term model for his partial set theory without extentionality.

\section{A term universe}

Let $\mathcal{N} \equiv\left\langle N ; \mathcal{L}_{\mathcal{N}}\right\rangle$ be any classical universe of set theory (e.g. a model of $Z F$; at least we require such an universe to be extensional). We naturally consider it as a $\mathcal{L}^{ \pm}$-structure by setting $\notin_{\mathcal{N}}:=N^{2} \backslash \in_{\mathcal{N}}$ and $\neq_{\mathcal{N}}:=N^{2} \backslash=_{\mathcal{N}}$ (so in which ' $\in$ ' \& ' $\notin$ ' are really classical negation of each other, as well as ' $=$ ' \& ' $\neq$ ', and we assume further that $=_{\mathcal{N}}$ is the real equality on $N$ ). Now let $\mathcal{L}_{\tau}^{ \pm}[N]$ be the natural extension of $\mathcal{L}^{ \pm}$obtained by using an abstraction operator ' $\{\mid\}$ ' and by considering each element in $N$ as a constant. Formally, the terms and formulae of $\mathcal{L}_{\tau}^{ \pm}[N]$ are defined inductively by the following rules:

1. Any variable is a term, as well as any constant $n$ in $N$.

2. If $\zeta$ and $\xi$ are terms, then ' $\zeta \in \xi$ ', ' $\zeta \notin \xi$ ', ' $\zeta=\xi$ ', ' $\zeta \neq \xi$ ' are formulae.

3. If $\varphi$ and $\psi$ are formulae and $x$ is a variable, then ' $\varphi \vee \psi$ ', ' $\varphi \wedge \psi$ ', ' $\forall x \varphi$ ', ' $\exists x \varphi$ ' are formulae.

4. If $\varphi$ is a formula, so is ' $\neg \varphi$ '.

5. If $\varphi$ is a formula, then ' $\{x \mid \varphi\}$ ' is a term.

The positive terms and formulae in $\mathcal{L}_{\tau}^{ \pm}[N]$ are those obtained without using rule 4 . The weak negation $\bar{\varphi}$ of such a positive formula $\varphi$ is defined naturally as in $\mathcal{L}^{ \pm}$by considering terms as variables. The reader should notice here that a positive formula in this extended language might not be positive in $\mathcal{L}^{ \pm}$, at least for its natural interpretation pretending that $E X T$ and $C O M P$ are fulfilled.

EXAMPLE 1.

Set $\xi:=\{x \mid x \in x\}$ and consider the positive $\mathcal{L}_{\tau}^{ \pm}$-formula ' $\xi \in \xi$ '. Its natural translation in $\mathcal{L}^{ \pm}$is ' $\exists y(y \in y \wedge \forall x(x \in y \leftrightarrow x \in x) \wedge \forall x(x \notin y \leftrightarrow x \notin x))$ ', which is not positive in $\mathcal{L}^{ \pm}$(not even bounded positive).

However, it is worth observing that the projective lemma remains true for positive $\mathcal{L}_{\tau}^{ \pm}[N]$-formulae as soon as the surjective morphism involved 
preserves terms, as it can be easily checked, since terms represent nothing but distinguished elements in any model. ${ }^{6}$

Now what we call a constant term is either a constant or a term of the form ' $\{x \mid \varphi(x)\}$ '. Here is such a term:

EXAMPLE 2 .

Set $\xi(y):=\{x \mid y \in y\}$ and let $\varphi(x)$ be the $\mathcal{L}_{\tau}^{ \pm}$-formula ' $\forall y(\xi(y) \in x)$ '. Then $\zeta:=\{x \mid \varphi(x)\}$ is a constant positive $\mathcal{L}_{\tau}^{ \pm}$-term.

Note that if $\left\{x \mid \varphi\left(x, y_{1}, \ldots, y_{k}\right)\right\}$ is any (positive) term and if $\zeta_{1}, \ldots, \zeta_{k}$ are any constant (positive) terms, then $\left\{x \mid \varphi\left[x, \zeta_{1}, \ldots, \zeta_{k}\right]\right\}$ is actually a constant (positive) term. Some special constant positive terms will play a particular role in our construction, so let us define the set of posterms over $N$ recursively as follows:

- Each $n \in N$ is a primitive posterm over $N$;

- If $t_{1}, \ldots, t_{k}$ are posterms over $N$ and if $\varphi\left(x, y_{1}, \ldots, y_{k}\right)$ is a positive $\mathcal{L}^{ \pm}$-formula, then ' $\left\{x \mid \varphi\left[x, t_{1}, \ldots, t_{k}\right]\right\}$ ' is a posterm over $N$.

Thus any posterm is clearly a constant positive term, but the converse is false as, for instance, the term $\zeta$ defined in example 2 shows. Notice by the way that example 1 proves actually that even the natural translation in $\mathcal{L}^{ \pm}$ of a formula such as ' $\varphi[\xi]$ ', where $\varphi(x)$ is positive $\underline{\text { in } \mathcal{L}^{ \pm}}$and $\xi$ is a posterm, might not be positive in $\mathcal{L}^{ \pm}$either.

From now on, we assume that the positive constant terms have been coded in the metatheory, in such a way that if $\ulcorner\zeta\urcorner$ denotes the code of $\zeta$, then $\ulcorner n\urcorner=n$ for any $n \in N$. So let $M$ be the set of those codes and $M^{*}$ the subset of $M$ corresponding to the posterms (thus $N \subseteq M^{*} \subseteq M$ ).

\section{Setting up $M$ as a $\mathcal{L}^{ \pm}$-structure}

We define a sequence $\left(\mathcal{M}_{\alpha} \mid \alpha \in O n\right)$ of $\mathcal{L}_{\tau_{+}}^{ \pm}[N]$-structures as follows (' $\tau_{+}$' means that only positive terms will be interpreted):

-) The universe of $\mathcal{M}_{\alpha}$ is $M$, for all $\alpha$, and the interpretation of any constant $n \in N$ is ' $n$ ' itself;

-) The interpretation of the positive term $\left\{x \mid \varphi\left(x, y_{1}, \ldots, y_{k}\right)\right\}$ in $\mathcal{M}_{\alpha}$, for all $\alpha$ and for $y_{1}=\left\ulcorner\zeta_{1}\right\urcorner, \ldots, y_{k}=\left\ulcorner\zeta_{k}\right\urcorner$ is ' $\left\ulcorner\left\{x \mid \varphi\left[x, \zeta_{1}, \ldots, \zeta_{k}\right]\right\}\right\urcorner$ ';

\footnotetext{
${ }^{6}$ Naturally, we say that a surjective morphism $f: \mathcal{M} \rightarrow \mathcal{N}$ preserves terms if $f^{\prime}\left\{x \mid \varphi\left[x, m_{1}, \ldots, m_{k}\right]\right\}_{\mathcal{M}}=\left\{x \mid \varphi\left[x, f^{\prime} m_{1}, \ldots, f^{\prime} m_{k}\right]\right\}_{\mathcal{N}}$, for any $m_{1}, \ldots, m_{k} \in M$.
} 
-) For all $\alpha,=_{\mathcal{M}_{\alpha}}$ is the real equality in $M$ and $\neq_{\mathcal{M}_{\alpha}}$ is classical;

-) $\epsilon_{\mathcal{M}_{\alpha}}$ and $\notin_{\mathcal{M}_{\alpha}}$ are defined inductively:

- Initial Step:

$$
\begin{cases}\epsilon_{\mathcal{M}_{0}} & :=\epsilon_{\mathcal{N}} \cup M \times(M \backslash N) \\ \notin_{\mathcal{M}_{0}}:=M^{2} \backslash \epsilon_{\mathcal{N}}\end{cases}
$$

- Successor Step:

$$
\begin{aligned}
& \text { If } n \in N \text {, then }\left\{\begin{array}{lll}
m \in_{\mathcal{M}_{\beta+1}} n & : \Leftrightarrow & m \in N \wedge m \in_{\mathcal{N}} n \\
m \notin_{\mathcal{M}_{\beta+1}} n & : \Leftrightarrow \quad\left(m \in N \wedge m \notin_{\mathcal{N}} n\right) \vee m \in(M \backslash N)
\end{array}\right. \\
& \text { If } n=\ulcorner\{x \mid \varphi(x)\}\urcorner \text {, then }\left\{\begin{array}{lll}
m \in_{\mathcal{M}_{\beta+1}} n & : \Leftrightarrow & \mathcal{M}_{\beta} \models \varphi[m] \\
m \notin_{\mathcal{M}_{\beta+1}} n & : \Leftrightarrow & \mathcal{M}_{\beta} \models \bar{\varphi}[m]
\end{array}\right.
\end{aligned}
$$

- Limit Step:

$$
\left\{\begin{array}{l}
\epsilon_{\mathcal{M}_{\lambda}}:=\bigcap_{\alpha<\lambda} \epsilon_{\mathcal{M}_{\alpha}} \\
\notin_{\mathcal{M}_{\lambda}}:=\bigcap_{\alpha<\lambda} \notin_{\mathcal{M}_{\alpha}}
\end{array}\right.
$$

An easy induction shows that, for all $\alpha \in O n, \epsilon_{\mathcal{M}_{\alpha}} \cap N^{2}=\epsilon_{\mathcal{N}}, \notin_{\mathcal{M}_{\alpha}} \cap N^{2}=$ $\notin_{\mathcal{N}}$ and $N$ is $\in$-initial in $\mathcal{M}_{\alpha} \cdot{ }^{7}$ Of course, $N$ is not $\notin$-initial since $M \backslash N \times$ $N \subseteq \notin_{\mathcal{M}_{\alpha}}$ for all $\alpha \in O n$. The next lemma tells us that $\left(\mathcal{M}_{\alpha} \mid \alpha \in O n\right)$ is a descending sequence of $P d \mathcal{L}^{ \pm}$-structures on $M$ :

\section{LEMMA.}

(i) $\forall \alpha \forall \beta \leqslant \alpha, \quad \epsilon_{\mathcal{M}_{\alpha}} \subseteq \epsilon_{\mathcal{M}_{\beta}} \quad \& \quad \notin_{\mathcal{M}_{\alpha}} \subseteq \notin_{\mathcal{M}_{\beta}}$;

(ii) $\forall \alpha, \quad \mathcal{M}_{\alpha} \models P d$-case.

Proof. The proof goes by induction on $\alpha \in O n$.

(i) If $\alpha$ is a limit ordinal or 0 , then the property is obviously true by definition. So let $\alpha=\beta+1$ and let the property be true for all $\gamma \leqslant \beta$; that means actually that the identity map on $M$ is a $\mathcal{L}^{ \pm}$-epimorphism from $\mathcal{M}_{\delta}$ to $\mathcal{M}_{\gamma}$, for all $\delta \leqslant \gamma \leqslant \beta$, and clearly, the terms are preserved too (see the definition of their interpretations). Now suppose that $m \in_{\mathcal{M}_{\beta+1}} n$ and let us check that this implies that $m \in_{\mathcal{M}_{\beta}} n$. If $n \in N$, that is obvious. Assume then that $n=\ulcorner\{x \mid \varphi(x)\}\urcorner$. Hence, by definition, we have $\mathcal{M}_{\beta} \models \varphi[m]$, and by using the (extended) "projective lemma", it follows that $\mathcal{M}_{\gamma} \models \varphi[m]$, for all

${ }^{7} N$ is said to be $R$-initial in $\left\langle M ; R_{\mathcal{M}}\right\rangle$ if $N \subseteq M$ and $\forall n \in N, \forall m \in M, m R_{\mathcal{M}} n \Rightarrow$ $m \in N$. 
$\gamma \leqslant \beta$. Therefore, $m \in_{\mathcal{M}_{\gamma+1}} n$, for all $\gamma<\beta$. So, when $\beta \neq 0$, we have:

$$
\epsilon_{\mathcal{M}_{\beta+1}} \subseteq \bigcap_{\gamma<\beta} \epsilon_{\mathcal{M}_{\gamma+1}} \subseteq \bigcap_{\gamma<\beta} \epsilon_{\mathcal{M}_{\gamma}}
$$

That shows that $\epsilon_{\mathcal{M}_{\beta+1}} \subseteq \epsilon_{\mathcal{M}_{\beta}}$. Of course, this is true as well if $\beta=0$ as the definition of $\in_{\mathcal{M}_{0}}$ shows. A symmetric argument applies to ' $\notin$ '.

(ii) Since ' $f$ ' is classical at each step, we focus on the ' $\in$ ' part. Let the property be true for all $\beta<\alpha$.

- $\alpha=0$ :

Then $\in_{\mathcal{M}_{0}} \cup \notin_{\mathcal{M}_{0}}=M^{2}$ by definition;

- $\alpha=\beta+1$ :

Let $n, m \in M$. Assume first that $n=\ulcorner\{x \mid \varphi(x)\}\urcorner$. Then by induction hypothesis, we know that $\mathcal{M}_{\beta} \models \varphi[m] \vee \bar{\varphi}[m]$, which means exactly that $m \in_{\mathcal{M}_{\beta+1}} n$ or $m \notin_{\mathcal{M}_{\beta+1}} n$. Now, if $n \in N$, this follows directly from the definition of ' $\in$ ' \& ' $\notin$ ' in $\mathcal{M}_{\beta+1}$.

- $\alpha=\lambda$ limit :

Suppose $\neg\left(m \in_{\mathcal{M}_{\lambda}} n\right)$. Then there exists $\beta_{0}<\lambda$ such that $\neg\left(m \in_{\mathcal{M}_{\beta_{0}}} n\right)$. Whence $m \notin_{\mathcal{M}_{\beta_{0}}} n$, by induction hypothesis, and this implies that $m \notin_{\mathcal{M}_{\lambda}} n$. Otherwise, there would exist $\gamma_{0}$ such that $\beta_{0}<\gamma_{0}<\lambda$ and $\neg\left(m \notin_{\mathcal{M}_{\gamma_{0}}} n\right)$. Then, again by induction hypothesis, this would imply that $m \in_{\mathcal{M}_{\gamma_{0}}} n$, which is impossible as $\neg\left(m \in_{\mathcal{M}_{\beta_{0}}} n\right)$ and $\in_{\mathcal{M}_{\gamma_{0}}} \subseteq \in_{\mathcal{M}_{\beta_{0}}}$ (by property (i)).

In view of this lemma, there does exist a least ordinal $\delta$ such that both $\epsilon_{\mathcal{M}_{\delta+1}}=\epsilon_{\mathcal{M}_{\delta}} \& \notin_{\mathcal{M}_{\delta+1}}=\notin_{\mathcal{M}_{\delta}}$. Then $\mathcal{M}_{\delta}$ does satisfy comprehension as the next theorem states:

\section{THEOREM 3.}

For any positive $\mathcal{L}_{\tau}^{ \pm}[N]$-formula $\varphi$,

$$
\mathcal{M}_{\delta} \models \forall y_{1}, \ldots, y_{k} \forall z\left\{\begin{aligned}
& z \in\left\{x \mid \varphi\left(x, y_{1}, \ldots, y_{k}\right)\right\} \leftrightarrow \varphi\left[z, y_{1}, \ldots, y_{k}\right] \\
& z \notin\left\{x \mid \varphi\left(x, y_{1}, \ldots, y_{k}\right)\right\} \leftrightarrow \bar{\varphi}\left[z, y_{1}, \ldots, y_{k}\right]
\end{aligned}\right.
$$

Proof. Let $\varphi\left(x, y_{1}, \ldots, y_{k}\right)$ be a positive $\mathcal{L}_{\tau}^{ \pm}[N]$-formula and let $\left\ulcorner\zeta_{1}\right\urcorner, \ldots$, $\left\ulcorner\zeta_{k}\right\urcorner \in M$. Now set $n:=\left\ulcorner\left\{x \mid \varphi\left[x, \zeta_{1}, \ldots, \zeta_{k}\right]\right\}\right\urcorner$. Then,

$$
m \in_{\mathcal{M}_{\beta}} n \Leftrightarrow m \in_{\mathcal{M}_{\beta+1}} n \Leftrightarrow \mathcal{M}_{\beta} \models \varphi\left[m,\left\ulcorner\zeta_{1}\right\urcorner, \ldots,\left\ulcorner\zeta_{k}\right\urcorner\right]
$$

and similarly for ' $\notin$ ' but with $\bar{\varphi}$ instead of $\varphi$. 
Thus $\mathcal{M}_{\delta} \models C O M P$. But unfortunately, $\mathcal{M}_{\delta} \not \models E X T$.

EXAMPLE 3.

Let $\zeta:=\{x \mid x=x\}$ and $\xi:=\{x \mid \zeta=\zeta\}$. Then it is easy to see that, for all $\alpha,[\ulcorner\zeta\urcorner]_{\mathcal{M}_{\alpha}}^{+}=[\ulcorner\xi\urcorner]_{\mathcal{M}_{\alpha}}^{+}=M$ and $[\ulcorner\zeta\urcorner]_{\mathcal{M}_{\alpha}}^{-}=[\ulcorner\xi\urcorner]_{\mathcal{M}_{\alpha}}^{-}=\emptyset$, but $\ulcorner\zeta\urcorner \neq\ulcorner\xi\urcorner$ yet!

So we have here a situation whereby the technique described in section 4 could be useful.

\section{Recovering extensionality}

We now call in theorem 2 required for that job. Let $\sim$ be the finest bisimulation as provided by the theorem. Our final model is $\widetilde{\mathcal{M}}_{\delta}{ }^{8}$ So it satisfies $E X T$, but what about $C O M P$ now? It turns out that a (slightly?) weaker version remains:

THEOREM 4.

$\widetilde{\mathcal{M}}_{\delta} \models C O M P^{b}$

where $C O M P^{b}: \equiv\left\{\begin{array}{l}\text { For any positive } \mathcal{L}^{ \pm} \text {-formula } \varphi, \\ \exists y \forall x((x \in y \rightarrow \varphi) \wedge(x \notin y \rightarrow \bar{\varphi})) \quad(y \text { not free in } \varphi)\end{array}\right.$

Proof. Observe that ' $\exists y \forall x((x \in y \rightarrow \varphi) \wedge(x \notin y \rightarrow \bar{\varphi}))$ ' is a $\operatorname{BP} \mathcal{L}^{ \pm}$formula whenever $\varphi$ is positive in $\mathcal{L}^{ \pm}$. Then, as noticed in section 4 , such a formula is preserved under a bisimulation.

Thus implications in the other direction are lost. Nevertheless, this version of comprehension really makes sense in the $P d$-case, as the reader should easily allow himself to be convinced by drawing a picture. Its translation in Pac is the following:

$C O M P^{b}: \equiv\left\{\begin{array}{c}\text { For any } \mathcal{L} \text {-formula } \varphi \text { in the implicationless fragment of Pac, } \\ \exists y \forall x(x \in y \rightleftharpoons \varphi) \quad(y \text { not free in } \varphi)\end{array}\right.$

However, as that clearly appears in the formulation above, $C O M P^{b}$ is not compatible with EXT any more. Indeed, it is no longer provable using $E X T$ that, for any given formula $\varphi$, there is only one set $y$ provided by $C O M P^{b}$. It follows that the natural interpretation of non-constant positive terms has been destroyed by the bisimulation: for $\ulcorner a\urcorner \sim\ulcorner b\urcorner$, we are not able to prove that $\ulcorner\{x \mid \varphi[x, a, \ldots]\}\urcorner \sim\ulcorner\{x \mid \varphi[x, b, \ldots]\}\urcorner$. Furthermore, although each set in $M \backslash N$ (being some $\ulcorner\{x \mid \varphi\}\urcorner$ ) is still designated by a

\footnotetext{
${ }^{8}$ We would just remind the reader that $=_{\overline{\mathcal{M}}_{\delta}}$ is nothing but $\sim$ ( equality on $\mathcal{M}_{\delta}$ ).
} 
positive $\mathcal{L}_{\tau}^{ \pm}[N]$-formula $\varphi$, the existence of such a set in $\widetilde{\mathcal{M}}_{\delta}$ is not either provided by the $\varphi$-instance of the current comprehension schema in $\widetilde{\mathcal{M}}_{\delta}$, i.e. $C O M P^{b}$, since only positive $\mathcal{L}^{ \pm}$-formulae are allowed in it. Hence the model we get in this way is no longer a pure "strong" ${ }^{9}$ term model as $\mathcal{M}_{\delta}$ was. In other words, many sets outside $N$ are quite artificial with regard to our formulation of comprehension in $\widetilde{\mathcal{M}}_{\delta}$. But this can be overcome if we only take $M^{*}$ into account. Indeed, any $\left\ulcorner\left\{x \mid \varphi\left[x, t_{1}, \ldots, t_{k}\right]\right\}\right\urcorner \in M^{*}$ satisfies the $\varphi\left(x, y_{1}, \ldots, y_{k}\right)$-instance of $C O M P^{b}$, for $y_{1}=\left\ulcorner t_{1}\right\urcorner, \ldots, y_{k}=\left\ulcorner t_{k}\right\urcorner$, as the definition of $M^{*}$ ensures that $\varphi\left(x, y_{1}, \ldots, y_{k}\right)$ is a positive $\mathcal{L}^{ \pm}$-formula. So a more "natural" model $\widetilde{\mathcal{M}}_{\delta}^{*}$ can be obtained by reiterating the construction described in $6.2 \& 6.3$ but with $M^{*}$ instead of $M$.

On the other hand, as expected, our construction does not change the meaning of the primitive symbols in $\mathcal{N}$, which really appears as an $\in$-initial and classical part of $\widetilde{\mathcal{M}}_{\delta}$ (and of $\widetilde{\mathcal{M}}_{\delta}^{*}$ as well). Perhaps this requires some explanations:

We already know that $\mathcal{N}$ is an $\in$-initial and classical part of $\mathcal{M}_{\delta}$ with $\epsilon_{\mathcal{M}_{\delta}} \cap N^{2}=\epsilon_{\mathcal{N}} \quad \& \quad \notin_{\mathcal{M}_{\delta}} \cap N^{2}=\notin_{\mathcal{N}}$. So it suffices to prove that $\sim \cap N^{2}$ is exactly $=_{\mathcal{N}}$. Recalling the proof of theorem 2, we have to show that $\sim_{\alpha} \cap N^{2}$ is $=_{\mathcal{N}}$, for all $\alpha \in O n$. This goes by induction. Of course, as $\mathcal{N}$ is supposed to be a classical model of some set theory, we assume that it is extensional (in its classical meaning), and that will do the job. To see that, suppose that $\sim_{\alpha} \cap N^{2}$ is $=_{\mathcal{N}}$ and recall that:

$$
x \sim_{\alpha+1} y \Leftrightarrow\left([x]_{\mathcal{M}_{\delta}}^{+}\right)^{\sim_{\alpha}}=\left([y]_{\mathcal{M}_{\delta}}^{+}\right)^{\sim_{\alpha}} \&\left([x]_{\mathcal{M}_{\delta}}^{-}\right)^{\sim_{\alpha}}=\left([x]_{\mathcal{M}_{\delta}}^{-}\right)^{\sim_{\alpha}}
$$

Now let $x, y \in N$. As $\mathcal{N}$ is $\in$-initial in $\mathcal{M}_{\delta}$ and $\epsilon_{\mathcal{M}_{\delta}} \cap N^{2}=\epsilon_{\mathcal{N}}$, we have $[x]_{\mathcal{M}_{\delta}}^{+}=[x]_{\mathcal{N}}^{+} \subseteq N$, and similarly for $y$. Thus, $x \sim_{\alpha+1} y \Rightarrow\left([x]_{\mathcal{N}}^{+}\right)^{\sim_{\alpha}}=$ $\left([y]_{\mathcal{N}}^{+}\right)^{\sim_{\alpha}}$. By transitivity of $\sim_{\alpha}$, this last equality can be reformulated more expressively as follows:

$$
[x]_{\mathcal{N}}^{+} \subseteq\left([y]_{\mathcal{N}}^{+}\right)^{\sim_{\alpha}} \quad \& \quad[y]_{\mathcal{N}}^{+} \subseteq\left([x]_{\mathcal{N}}^{+}\right)^{\sim_{\alpha}}
$$

Now, by induction hypothesis, this amounts to:

$$
[x]_{\mathcal{N}}^{+} \subseteq[y]_{\mathcal{N}}^{+} \quad \& \quad[y]_{\mathcal{N}}^{+} \subseteq[x]_{\mathcal{N}}^{+}
$$

Therefore, $x \sim_{\alpha+1} y \Rightarrow[x]_{\mathcal{N}}^{+}=[y]_{\mathcal{N}}^{+} \Rightarrow x={ }_{\mathcal{N}} y$, assuming that $\mathcal{N}$ is extensional, and thus $\sim_{\alpha+1}$ is still $=_{\mathcal{N}}$ on $N$.

\footnotetext{
${ }^{9}$ In the sense that the terms in $\mathcal{M}_{\delta}$ are defined by means of $\mathcal{L}_{\tau}^{ \pm}[N]$-formulae, so within a richer language involving terms themselves.
} 
The reader might wonder what the difference relation looks like in our model. By definition of $\widetilde{\mathcal{M}}_{\delta}$, we have:

$$
x \neq_{\widetilde{\mathcal{M}}_{\delta}} y \Leftrightarrow \exists x^{\prime} \sim x, \exists y^{\prime} \sim y: x^{\prime} \neq_{\mathcal{M}_{\delta}} y^{\prime}
$$

As $\widetilde{\mathcal{M}}_{\delta} \models P d$-case and ' $\neq$ ' is classical in $\mathcal{M}_{\delta}$, we get:

$$
x \neq_{\widetilde{\mathcal{M}}_{\delta}} y \Leftrightarrow \neg(x \sim y) \vee\left(x \sim y \wedge\left|(\{x\})^{\sim}\right|>1\right)
$$

(where $|A|$ denotes the cardinal number of $A$ ) and it turns out that $m \neq \overline{\widetilde{M}}_{\delta} m$, for any $m \in M \backslash N$. Indeed, fix $n_{0} \in N$ and let $m:=\ulcorner\{x \mid \varphi\}\urcorner \in M N$. Now set $m^{\prime}:=\left\ulcorner\left\{x \mid \varphi \wedge n_{0}=n_{0}\right\}\right\urcorner$. Thus, it is fairly clear that $m$ and $m^{\prime}$ have the same extensions in $\mathcal{M}_{\delta}$, so $m \sim m^{\prime}$, but $m \neq m^{\prime}$.

\section{Acknowledgments}

I would like to express my gratitude to Roland Hinnion for launching me on this subject and giving much advice for its study.

\section{References}

[1] Avron, A., 'Natural 3-valued logics - characterization and proof theory', The Journal of Symbolic Logic, 56: 276-294, 1991.

[2] Avron, A., 'On an implication connective of RM', Notre Dame Journal of Formal Logic, 27: 201-209, 1986.

[3] Batens, D., 'Paraconsistent extensional propositional logics', Logique et Analyse, 90-91: 195-234, 1980.

[4] Batens, D., and K. De ClercQ, 'A rich paraconsistent extension of full positive logic' (2000), to appear.

[5] Brady, R. T., 'The consistency of the axioms of abstraction and extensionality in a three-valued logic', Notre Dame Journal of Formal Logic, 12: 447-453, 1991.

[6] da Costa, N. C. A., and O. A. S. Bueno, 'Paraconsistency: towards a tentative interpretation', Theoria, vol 16, 40: 119-145, 2001.

[7] Esser, O., 'Interprétations mutuelles entre une théorie positive des ensembles et une extension de la théorie de Kelley-Morse', Ph.D. thesis, Université Libre de Bruxelles, 1997, unpublished.

(available at http://homepages.ulb.ac.be/ oesser)

[8] Esser, O., 'Inconsistency of the axiom of choice with the positive theory GPK $K_{\infty}^{+}$', The Journal of Symbolic Logic, 65: 1911-1916, 2000. 
[9] Forti, M., and R. Hinnion, 'The consistency problem for positive comprehension principles', The Journal of Symbolic Logic, 54: 1401-1418, 1989.

[10] Gilmore, P. C., 'The consistency of partial set theory without extensionality', Proceedings of Symposia in Pure Mathematics, 13: 147-153, 1974.

[11] Hinnion, R., 'Naive set theory with extensionality in partial logic and in paradoxical logic', Notre Dame Journal of Formal Logic, 35: 15-40, 1994.

[12] Hinnion, R., 'About the coexistence of classical sets with non-classical ones: a survey', Logic and Logical Philosophy, this issue.

[13] Restall, G., 'A note on naive set theory in LP', Notre Dame Journal of Formal Logic, 33: 422-432, 1992.

THIERRY LIBERT

Service de Logique

Département de Mathématiques

Université Libre de Bruxelles

CP211, Boulevard du Triomphe

1050 Brussels

Belgium

tlibert@ulb.ac.be 